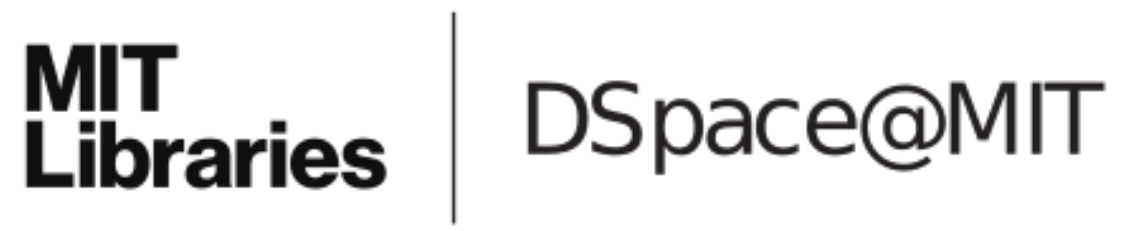

\author{
MIT Open Access Articles
}

Electromagnetic interaction of arbitrary radialdependent anisotropic spheres and improved invisibility for nonlinear-transformation-based cloaks

The MIT Faculty has made this article openly available. Please share how this access benefits you. Your story matters.

Citation: Qiu, Cheng-Wei et al. "Electromagnetic interaction of arbitrary radial-dependent anisotropic spheres and improved invisibility for nonlinear-transformation-based cloaks."

Physical Review E 80.1 (2009): 016604. (C) 2010 The American Physical Society.

As Published: http://dx.doi.org/10.1103/PhysRevE.80.016604

Publisher: American Physical Society

Persistent URL: http://hdl.handle.net/1721.1/51339

Version: Final published version: final published article, as it appeared in a journal, conference proceedings, or other formally published context

Terms of Use: Article is made available in accordance with the publisher's policy and may be subject to US copyright law. Please refer to the publisher's site for terms of use. 


\title{
Electromagnetic interaction of arbitrary radial-dependent anisotropic spheres and improved invisibility for nonlinear-transformation-based cloaks
}

\author{
Cheng-Wei Qiu, ${ }^{1,2, *}$ Andrey Novitsky, ${ }^{3}$ Hua Ma, ${ }^{4}$ and Shaobo $\mathrm{Qu}^{4}$ \\ ${ }^{1}$ Research Laboratory of Electronics, Massachusetts Institute of Technology, \\ 77 Massachusetts Avenue, Cambridge, Massachusetts 02139, USA \\ ${ }^{2}$ Department of Electrical and Computer Engineering, National University of Singapore, Kent Ridge, Singapore 119620, Singapore \\ ${ }^{3}$ Department of Theoretical Physics, Belarusian State University, Nezavisimosti Avenue 4, 220050 Minsk, Belarus \\ ${ }^{4}$ The College of Science, Air Force University of Engineering, Xi'an 710051, China
}

(Received 8 April 2009; published 27 July 2009)

\begin{abstract}
An analytical method of electromagnetic wave interactions with a general radially anisotropic cloak is established. It is able to deal with arbitrary parameters $\left[\varepsilon_{r}(r), \mu_{r}(r), \varepsilon_{t}(r)\right.$, and $\left.\mu_{t}(r)\right]$ of a radially anisotropic inhomogeneous shell. The general cloaking condition is proposed from the wave relations, in contrast to the method of transformation optics. Spherical metamaterial cloaks with improved invisibility performance are achieved with optimal nonlinearity in transformation and core-shell ratio.
\end{abstract}

DOI: 10.1103/PhysRevE.80.016604

PACS number(s): 41.20.Jb, 42.25.Gy, 42.79.Dj

\section{INTRODUCTION}

Coordinate transformation [1-4] for the design process of the cloaking devices has received great attention. The cylindrical/spherical cloaking idea proposed by Pendry [1] is to employ radial anisotropic materials whose parameters are determined from the topological variation between the original and transformed spaces, based on the invariance of Maxwell's equations throughout a specific coordinate transformation [3]. The idea of cylindrical cloaking was confirmed by analytical/full-wave methods [5-7] and verified by an experiment using artificial metamaterials with inclusions of metallic split-ring resonators (SRRs) [8]. So far, significant progress has been made on the study of cylindrical invisibility cloaks. It reveals that the simplified parameters for cylindrical cloaking still allow wave interactions with the cloaked object [9] and the invisibility performance of a cylindrical cloak is very sensitive to the geometrical perturbation of its interior boundary [10], which can be both fixed by introducing perfect electric conducting (PEC) or perfectly magnetic conducting (PMC) linings onto the inner surface of the shell $[4,11]$. Since it is challenging to synthesize the magnetic response in optical regime, nonmagnetic cylindrical cloaks have been proposed by using quadratic transformation [12] and the general high-order transformation for nonmagnetic cylindrical cloaks in optical frequency is addressed more recently [13]. Nevertheless, it is still difficult to realize the position-dependent cylindrical cloak due to the limited resource of natural materials exhibiting radial anisotropy [14]. In view of this, Cai et al. proposed a multilayered cylindrical cloak by dividing the original position-dependent cloak into many thin coatings in which the material parameters become homogeneous [15]. Furthermore, the cylindrical cloak has been theoretically realized by a concentric cylinder of isotropic homogeneous multilayers [16]. Arbitrary-shaped twodimensional (2D) cloaks have been investigated theoretically and numerically [17-19].

\footnotetext{
*cwq@mit.edu
}

However, for spherical invisibility cloaks there are still a lot of unknowns to be explored because of the complexity in analysis and simulation of scattering properties. Anisotropic and position-dependent ideal spherical cloaks based on the linear transformation were suggested by Pendry [1], and it has been shown that spherical cloaks are less sensitive to the perturbation than cylindrical cloaks [10], which is mathematically proven [20]. There are several main streams studying linear first-order spherical invisibility cloaks, whose the required materials and the corresponding methods are distinct. The first approach is the classic cloak [1], which is linear, anisotropic, and inhomogeneous. In this connection, explicitly electromagnetic fields have been formulated [21] and it is further confirmed that the wave cannot interact with the concealed object [22]. The second is to utilize a homogeneous anisotropic metamaterial cover to achieve electromagnetic invisibility [23] via the core-shell system. The third is the implementation of isotropic plasmonic materials as the cloak based on cancellation scheme [24,25]. The fourth is to substitute the Pendry's classic cloak with alternating thin multishells, and each shell is homogeneous and isotropic [26]. Each approach mentioned above has its own advantages and restrictions. For instance, the first approach [1] requires higher complexity in material parameters, and the analysis is situated toward a particular anisotropy ratio, which is addressed in [26]. The second approach [23] removes the requirement of material inhomogeneity, in which parameters are position independent. However, its cloaking property is quite reliant on the core-shell ratio, and the same feature is possessed by the third approach (cancellation scheme). The fourth method has less restrictions on materials but needs a lot of coatings that are sufficiently thin compared with the wavelength. The high-order term in the refractive index of an inhomogeneous spherical lens is discussed and its possibility of realizing a spherical cloak without parametric singularity is addressed [27]. The critical material singularity is thus transformed into the geometrical singularity, which is less demanding [28].

In this paper, a more general high-order nonlinear transformation will be considered for spherical cloaks. We first 


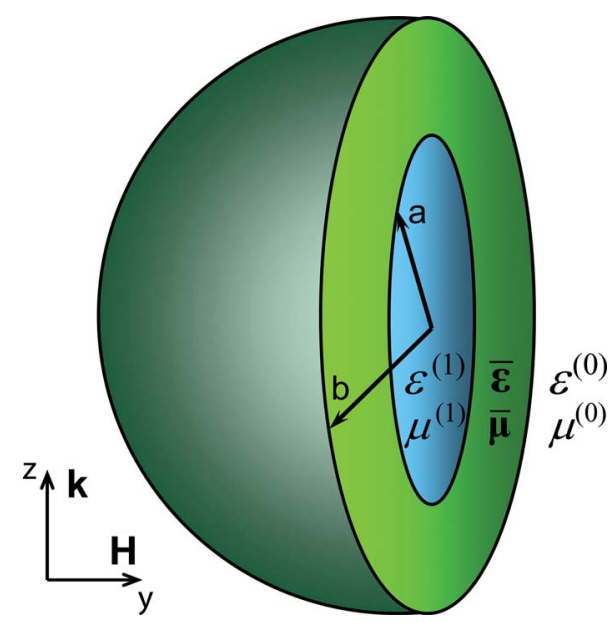

FIG. 1. (Color online) The geometry of the spherical cloak structure. Incident plane wave is propagating along $z$ direction and its electric field is polarized along $x$ direction. The subscripts 0 and 1 denote the parameters of the host and cloaked media, respectively. The anisotropic $\overline{\boldsymbol{\varepsilon}}$ and $\overline{\boldsymbol{\mu}}$ presents the parameters of the cloak shell.

propose a general algorithm to study the electromagnetic scattering by a particle coated by a radially inhomogeneous shell whose anisotropic parameters can be arbitrary. We discretize the shell into multiple spherical shells, each of which is homogeneous and anisotropic. Also, we propose a class of nonlinear transformation based (NTB) spherical cloaks, whose anisotropy ratio is position dependent and also too complicated to be treated by any mentioned methods. By utilizing the established general scattering algorithm, the invisibility performance and its dependence on the nonlinear transformation are investigated. Finally, the numerical results suggest a particular type of nonlinear spherical cloak providing better invisibility than Pendry's linear spherical cloak [1].

\section{SCATTERING ALGORITHM FOR A GENERAL RADIALLY ANISOTROPIC METAMATERIAL CLOAK}

Figure 1 illustrates the configuration of the cloak structure, i.e., the inner and outer radii are denoted by $a$ and $b$, respectively; innermost region is filled by an isotropic dielectric material characterized by $\varepsilon^{(1)}$ and $\mu^{(1)}$; intermediate region is occupied by a general spherical metamaterial cloak characterized by $\bar{\varepsilon}$ and $\overline{\boldsymbol{\mu}}$

$$
\overline{\boldsymbol{\varepsilon}}=\varepsilon_{r}(r) \mathbf{e}_{r} \otimes \mathbf{e}_{r}+\varepsilon_{t}(r) \overline{\boldsymbol{I}}_{t}, \quad \overline{\boldsymbol{\mu}}=\mu_{r}(r) \mathbf{e}_{r} \otimes \mathbf{e}_{r}+\mu_{t}(r) \overline{\boldsymbol{I}}_{t},
$$

where $\varepsilon_{r}$ and $\mu_{r}$ are the radial permittivity and permeability, $\varepsilon_{t}$ and $\mu_{t}$ are the transversal material parameters, $\overline{\boldsymbol{I}}_{t}=\overline{\boldsymbol{I}}-\mathbf{e}_{r}$ $\otimes \mathbf{e}_{r}=\mathbf{e}_{\theta} \otimes \mathbf{e}_{\theta}+\mathbf{e}_{\varphi} \otimes \mathbf{e}_{\varphi}$ is the projection operator onto the plane perpendicular to the vector $\mathbf{e}_{r}, \bar{I}$ is the unit threedimensional dyad, and unit vectors $\mathbf{e}_{r}, \mathbf{e}_{\theta}$, and $\mathbf{e}_{\varphi}$ are the basis vectors of the spherical coordinates.

In this section, the scattering theory of multilayer anisotropic spherical particles is provided and applied to study a cloak. We suppose that the arbitrary field distribution of the incident monochromatic wave interacts with the two-layer sphere.

Using the separation of variables, the solution of Maxwell's equations in spherical coordinates $(r, \theta, \varphi)$ can be presented as

$$
\mathbf{E}(r, \theta, \varphi)=F_{l m}(\theta, \varphi) \mathbf{E}(r), \quad \mathbf{H}(r, \theta, \varphi)=F_{l m}(\theta, \varphi) \mathbf{H}(r),
$$

where the designation $\mathbf{E}(r)$ means that the components of the electric field vector depend only on the radial coordinate $r$ as $E_{r}(r), E_{\theta}(r)$, and $E_{\varphi}(r)$ (however, the vector itself includes the angle dependence in the basis vectors), and the secondrank tensor in three-dimensional space $F_{l m}$ serves to separate the variables ( $l$ and $m$ are the integer numbers). It can be written as the sum of dyads,

$$
F_{l m}=Y_{l m} \mathbf{e}_{r} \otimes \mathbf{e}_{r}+\mathbf{X}_{l m} \otimes \mathbf{e}_{\theta}+\left(\mathbf{e}_{r} \times \mathbf{X}_{l m}\right) \otimes \mathbf{e}_{\varphi},
$$

where $Y_{l m}(\theta, \varphi)$ and $\mathbf{X}_{l m}(\theta, \varphi)$ are the scalar and vector spherical harmonics, the orthogonality of which has been well described in [29]. Tensor functions $F_{l m}$ are very useful because they completely describe the angle dependence of the spherical electromagnetic waves and satisfy the orthogonality conditions

$$
\int_{0}^{\pi} \int_{0}^{2 \pi} F_{l^{\prime} m^{\prime}}^{+}(\theta, \varphi) F_{l m}(\theta, \varphi) \sin \theta \mathrm{d} \theta \mathrm{d} \varphi=\bar{I} \delta_{l^{\prime} l} \delta_{m^{\prime} m},
$$

where the superscript + stands for the Hermitian conjugate.

From the commutation of $\overline{\boldsymbol{\varepsilon}}, \overline{\boldsymbol{\mu}}$, and $F_{l m}$, it follows that the electric and magnetic fields obey the set of ordinary differential equations

$$
\begin{gathered}
\mathbf{e}_{r}^{\times} \frac{d \mathbf{H}}{d r}+\frac{1}{r} \mathbf{e}_{r}^{\times} \mathbf{H}-\frac{i \sqrt{l(l+1)}}{r} \mathbf{e}_{\varphi}^{\times} \mathbf{H}=-i k_{0} \overline{\boldsymbol{\varepsilon}} \cdot \mathbf{E}, \\
\mathbf{e}_{r}^{\times} \frac{d \mathbf{E}}{d r}+\frac{1}{r} \mathbf{e}_{r}^{\times} \mathbf{E}-\frac{i \sqrt{l(l+1)}}{r} \mathbf{e}_{\varphi}^{\times} \mathbf{E}=i k_{0} \overline{\boldsymbol{\mu}} \cdot \mathbf{H},
\end{gathered}
$$

where $k_{0}=\omega / c$ is the wave number in vacuum, and $\omega$ denotes the circular frequency of the incident electromagnetic wave. Quantity $\mathbf{n}^{\times}$is called the tensor dual to the vector $\mathbf{n}$ [30]. It results in the vector product, if multiplied by a vector $\mathbf{a}$ as $\mathbf{n}^{\times} \mathbf{a}=\mathbf{n} \mathbf{a}^{\times}=\mathbf{n} \times \mathbf{a}$.

Equation (5) results from variable separation in Maxwell's equations, and includes two algebraic scalar equations; therefore, two field components, $H_{r}$ and $E_{r}$, can be expressed by means of the rest four components. This can be presented in terms of the matrix link between the total fields $\mathbf{H}=\mathbf{H}_{\mathrm{t}}+H_{r} \mathbf{e}_{r}$ and $\mathbf{E}=\mathbf{E}_{\mathrm{t}}+E_{r} \mathbf{e}_{r}$, and their tangential components $\mathbf{H}_{\mathrm{t}}$ and $\mathbf{E}_{\mathrm{t}}$, 


$$
\begin{gathered}
\left(\begin{array}{l}
\mathbf{H}(r) \\
\mathbf{E}(r)
\end{array}\right)=V(r)\left(\begin{array}{l}
\mathbf{H}_{\mathrm{t}}(r) \\
\mathbf{E}_{\mathrm{t}}(r)
\end{array}\right), \\
V(r)=\left(\begin{array}{cc}
\overline{\boldsymbol{I}} & \frac{\sqrt{l(l+1)}}{\mu_{r}(r) k_{0} r} \mathbf{e}_{r} \otimes \mathbf{e}_{\theta} \\
-\frac{\sqrt{l(l+1)}}{\varepsilon_{r}(r) k_{0} r} \mathbf{e}_{r} \otimes \mathbf{e}_{\theta} & \overline{\boldsymbol{I}}
\end{array}\right) .
\end{gathered}
$$

Excluding the radial components of the fields from Eq. (5), we arrive at a set of ordinary differential equations of the first order for the tangential components, which can be interconnected by a four-dimensional vector $\mathbf{W}(r)$ as

$$
\frac{d \mathbf{W}(r)}{d r}=i k_{0} M(r) \mathbf{W}(r),
$$

where

$$
\begin{gathered}
M=\left(\begin{array}{cc}
A & B \\
C & D
\end{array}\right), \quad \mathbf{W}=\left(\begin{array}{c}
\mathbf{H}_{\mathrm{t}} \\
\mathbf{E}_{\mathrm{t}}
\end{array}\right) \equiv\left(\begin{array}{c}
H_{\theta} \\
H_{\varphi} \\
E_{\theta} \\
E_{\varphi}
\end{array}\right), \\
A=D=\frac{i}{k_{0} r} \overline{\boldsymbol{I}}, \quad B=\varepsilon_{t}(r) \mathbf{e}_{r}^{\times}-\frac{l(l+1)}{\mu_{r}(r) k_{0}^{2} r^{2}} \mathbf{e}_{\varphi} \otimes \mathbf{e}_{\theta}, \\
C=-\mu_{t}(r) \mathbf{e}_{r}^{\times}+\frac{l(l+1)}{\varepsilon_{r}(r) k_{0}^{2} r^{2}} \mathbf{e}_{\varphi} \otimes \mathbf{e}_{\theta} .
\end{gathered}
$$

Since $\mathbf{H}_{\mathrm{t}}$ and $\mathbf{E}_{\mathrm{t}}$ are continuous at the spherical interface, they can be used for solving the scattering problem. Now, we analyze the situation of $r$-dependent permittivities and permeabilities, which arise from the spherical cloaking. Excluding the $\varphi$ components of fields from Eq. (7), we derive the differential equation of the second order for the vector $w_{\theta}$ $=\mathbf{e}_{\theta} \mathbf{W}=\left(H_{\theta}, E_{\theta}\right)$ :

$$
\begin{aligned}
w_{\theta}^{\prime \prime} & +\frac{2}{r} w_{\theta}^{\prime}-\left(\begin{array}{cc}
\frac{\varepsilon_{t}^{\prime}}{\varepsilon_{t}} & 0 \\
0 & \frac{\mu_{t}^{\prime}}{\mu_{t}}
\end{array}\right) w_{\theta}^{\prime}+\left[k_{0}^{2} \varepsilon_{t} \mu_{t}-\frac{1}{r}\left(\begin{array}{cc}
\frac{\varepsilon_{t}^{\prime}}{\varepsilon_{t}} & 0 \\
0 & \frac{\mu_{t}^{\prime}}{\mu_{t}}
\end{array}\right)\right. \\
& \left.-\frac{l(l+1)}{r^{2}}\left(\begin{array}{cc}
\frac{\varepsilon_{t}}{\varepsilon_{r}} & 0 \\
0 & \frac{\mu_{t}}{\mu_{r}}
\end{array}\right)\right] w_{w_{\theta}=0,}
\end{aligned}
$$

where the prime denotes the derivative with respect to $r$. Further we will apply one condition on the medium parameters, which is usually used for the spherical cloaks: $\varepsilon_{r}(r)$ $=\mu_{r}(r)$ and $\varepsilon_{t}(r)=\mu_{t}(r)$ due to the impedance matching. Then the equations for $H_{\theta}$ and $E_{\theta}$ coincide and can be written in the form

$$
w_{\theta}^{\prime \prime}+\left(\frac{2}{r}-\frac{\varepsilon_{t}^{\prime}}{\varepsilon_{t}}\right) w_{\theta}^{\prime}+\left[k_{0}^{2} \varepsilon_{t}^{2}-\frac{\varepsilon_{t}^{\prime}}{r \varepsilon_{t}}-\frac{l(l+1)}{r^{2}} \frac{\varepsilon_{t}}{\varepsilon_{r}}\right] w_{\theta}=0 .
$$

This equation can be solved analytically only in very few cases. As an example, we can offer a case of $\varepsilon_{t}=\mu_{t}=a_{1} / r$ and $\varepsilon_{r}=\mu_{r}=a_{2} / r$, where $a_{1,2}$ are arbitrary values. However, such dependencies do not provide the cloak properties. Another solvable case in Eq. (10) is just Pendry's cloak, that is, $\varepsilon_{t}$ $=\mu_{t}=b /(b-a)$ and $\varepsilon_{r}=\mu_{r} \varepsilon_{t}(r-a)^{2} / r^{2}$.

Although analytical solutions cannot be found for all situations, the general structure of solutions can be studied. The solution of two differential equations in second order [Eq. (9)] contains four integration constants $c_{1}, c_{2}, c_{1}^{\prime}$, and $c_{2}^{\prime}$. The constants can be joined together into a couple of vectors $\mathbf{c}_{1}$ $=c_{1} \mathbf{e}_{\theta}+c_{1}^{\prime} \mathbf{e}_{\varphi}$ and $\mathbf{c}_{2}=c_{2} \mathbf{e}_{\theta}+c_{2}^{\prime} \mathbf{e}_{\varphi} \cdot \varphi$ components of the field vectors $H_{\varphi}$ and $E_{\varphi}$ are expressed in terms of the $\theta$ components, which have been already determined. The relation between $\theta$ and $\varphi$ components follows from Eq. (7). Summing up both components, the resultant field can be presented as

$$
\mathbf{W}=S(r) \mathbf{C}, \quad S(r)=\left(\begin{array}{ll}
\eta_{1}(r) & \eta_{2}(r) \\
\zeta_{1}(r) & \zeta_{2}(r)
\end{array}\right), \quad \mathbf{C}=\left(\begin{array}{l}
\mathbf{c}_{1} \\
\mathbf{c}_{2}
\end{array}\right),
$$

where $\eta_{1,2}$ and $\zeta_{1,2}$ are the two-dimensional blocks of the matrix $S(r)$. $\left\{\eta_{1}, \zeta_{1}, \mathbf{c}_{1}\right\}$ and $\left\{\eta_{2}, \zeta_{2}, \mathbf{c}_{2}\right\}$ denote the first and second sets of the independent solution of Eq. (9), respectively. Therefore, the general solution can be decomposed into two terms as $\mathbf{W}=\mathbf{W}^{(1)}+\mathbf{W}^{(2)}$, where

$$
\mathbf{W}^{(1)}=\left(\begin{array}{l}
\mathbf{H}_{\mathrm{t} 1} \\
\mathbf{E}_{\mathrm{t} 1}
\end{array}\right)=\left(\begin{array}{l}
\eta_{1} \\
\zeta_{1}
\end{array}\right) \mathbf{c}_{1}, \quad \mathbf{W}^{(2)}=\left(\begin{array}{c}
\mathbf{H}_{\mathrm{t} 2} \\
\mathbf{E}_{\mathrm{t} 2}
\end{array}\right)=\left(\begin{array}{c}
\eta_{2} \\
\zeta_{2}
\end{array}\right) \mathbf{c}_{2} .
$$

Electric and magnetic fields of each independent wave are related by means of impedance tensor $\Gamma$ as $\mathbf{E}_{\mathrm{t} j}=\Gamma_{j} \mathbf{H}_{\mathrm{t} j}(j$ $=1,2)$. Thus, the impedance tensor equals

$$
\Gamma_{j}(r)=\zeta_{j}(r) \eta_{j}^{-1}(r) .
$$

Vectors $\mathbf{c}_{1}$ and $\mathbf{c}_{2}$ can be expressed by means of the known tangential electromagnetic field $\mathbf{W}(a)$ as $\mathbf{C}$ $=S^{-1}(a) \mathbf{W}(a)$. Thus Eq. (11) can be rewritten as follows

$$
\mathbf{W}(r)=\Omega_{a}^{r} \mathbf{W}(a), \quad \Omega_{a}^{r}=S(r) S^{-1}(a),
$$

where the evolution operator (transfer matrix) $\Omega_{a}^{r}$ connects tangential field components at two distinct spatial points, i.e., $r$ and $a$. One can obtain the complete solution of the fields $\mathbf{E}(\mathbf{r})$ and $\mathbf{H}(\mathbf{r})$ by summing over $l$ and $m$ in the subsequent tensor product of $F_{l m}(\theta, \varphi)$ describing angle dependence [Eq. (3)], matrix $V^{l}(r)$ restoring the fields with their tangential components [Eq. (6)], and tangential field vectors [Eq. (11)],

$$
\begin{aligned}
\left(\begin{array}{l}
\mathbf{H}(\mathbf{r}) \\
\mathbf{E}(\mathbf{r})
\end{array}\right)= & \sum_{l=0}^{\infty} \sum_{m=-l}^{l}\left(\begin{array}{cc}
F_{l m}(\theta, \varphi) & 0 \\
0 & F_{l m}(\theta, \varphi)
\end{array}\right) \\
& \times V^{l}(r)\left(\begin{array}{ll}
\eta_{1}^{l}(r) & \eta_{2}^{l}(r) \\
\zeta_{1}^{l}(r) & \zeta_{2}^{l}(r)
\end{array}\right)\left(\begin{array}{c}
\mathbf{c}_{1}^{l m} \\
\mathbf{c}_{2}^{l m}
\end{array}\right) .
\end{aligned}
$$

In general, the solutions cannot be studied in the closed form for nonlinear spherical cloaks. Therefore, the approximate method of numerical computations is applied. An inhomogeneous anisotropic spherical cloak $a<r<b$ is equally divided into $N$ homogeneous anisotropic spherical layers, 
i.e., replaced with a multilayer structure. The number of the layers strongly determines the accuracy of calculations. The $j$ th homogeneous shell is situated in the region between $r$ $=a_{j-1}$ and $r=a_{j}$, where $j=1, \ldots, N, a_{0}=a$, and $a_{N}=b$. Wave solution of the single homogeneous layer can be represented in the form of the evolution operator $\Omega_{a_{j-1}}^{a_{j}}$. The solution for the whole inhomogeneous shell is thus the subsequent product of the elementary evolution operators,

$$
\Omega_{a}^{b}=\Omega_{a_{N-1}}^{b} \cdots \Omega_{a_{1}}^{a_{2}} \Omega_{a}^{a_{1}} .
$$

The solution of Eq. (9) in one layer with constant permittivities $\varepsilon_{r}, \varepsilon_{t}$, and permeabilities $\mu_{r}, \mu_{t}$ is expressed by means of a couple of independent spherical functions $g_{\nu}^{(1)}$ and $g_{\nu}^{(2)}$,

$$
\left(\begin{array}{c}
H_{\theta}(r) \\
E_{\theta}(r)
\end{array}\right)=\left(\begin{array}{l}
g_{\nu_{1}}^{(1)}\left(k_{t} r\right) c_{1}+g_{\nu_{1}}^{(2)}\left(k_{t} r\right) c_{2} \\
g_{\nu_{2}}^{(1)}\left(k_{t} r\right) c_{1}^{\prime}+g_{\nu_{2}}^{(2)}\left(k_{t} r\right) c_{2}^{\prime}
\end{array}\right)
$$

where $\quad k_{t}=k_{0} \sqrt{\varepsilon_{t} \mu_{t}}, \quad \nu_{1}=\sqrt{l(l+1) \varepsilon_{t} / \varepsilon_{r}+1 / 4}-1 / 2, \quad \nu_{2}$ $=\sqrt{l(l+1) \mu_{t} / \mu_{r}+1 / 4}-1 / 2$, which applies to both uniaxial anisotropic and bianisotropic media [28,31,32]. Functions $g_{\nu}^{(1,2)}$ of the order of $\nu$ can be spherical Bessel functions, modified spherical Bessel functions, or spherical Hankel functions.

Blocks $\eta$ and $\zeta$ introduced in Eq. (11) are the tensors

$$
\begin{gathered}
\eta_{1,2}=g_{\nu_{1}}^{(1,2)} \mathbf{e}_{\theta} \otimes \mathbf{e}_{\theta}-\frac{i}{\mu_{t} k_{0} r} \frac{d\left(r g_{\nu_{2}}^{(1,2)}\right)}{d r} \mathbf{e}_{\varphi} \otimes \mathbf{e}_{\varphi}, \\
\zeta_{1,2}=g_{\nu_{2}}^{(1,2)} \mathbf{e}_{\theta} \otimes \mathbf{e}_{\varphi}+\frac{i}{\varepsilon_{t} k_{0} r} \frac{d\left(r g_{\nu_{1}}^{(1,2)}\right)}{d r} \mathbf{e}_{\varphi} \otimes \mathbf{e}_{\theta},
\end{gathered}
$$

which can be presented as two-dimensional matrices for computation purposes.

Now we turn to the scattering of electromagnetic waves from the cloaking structure depicted in Fig. 1. We suppose that electromagnetic fields $\mathbf{H}_{\text {inc }}(\mathbf{r})$ and $\mathbf{E}_{\text {inc }}(\mathbf{r})$ are incident on the coated spherical particle from air $\left(\varepsilon^{(0)}=1, \mu^{(0)}=1\right)$. Wave solutions in each of the $N$ layers can be written using the general solution equation [Eq. (15)], which is already known. Scattered field propagating in air can be presented by the superposition of diverging spherical waves, which are mathematically described by spherical Hankel functions of the first kind $h_{\nu}^{(1)}(x)$. Let us first introduce $\widetilde{\eta}$ and $\widetilde{\zeta}$, which correspond to the tensors $\eta$ and $\zeta$ in Eq. (18) when Hankel functions replace Bessel functions. Then we obtain the scattered fields

$$
\begin{aligned}
\left(\begin{array}{c}
\mathbf{H}_{\mathrm{sc}}(\mathbf{r}) \\
\mathbf{E}_{\mathrm{sc}}(\mathbf{r})
\end{array}\right)= & \sum_{l=0}^{\infty} \sum_{m=-l}^{l}\left(\begin{array}{cc}
F_{l m} & 0 \\
0 & F_{l m}
\end{array}\right) V_{\mathrm{sc}}^{l}(r)\left(\begin{array}{c}
I \\
\tilde{\Gamma}^{l}(r)
\end{array}\right) \\
& \times \tilde{\eta}^{l}(r)\left[\widetilde{\eta}^{l}(b)\right]^{-1} \mathbf{H}_{\mathrm{sc}}^{l m}(b),
\end{aligned}
$$

where $\widetilde{\Gamma}^{l}=\widetilde{\zeta}^{l}\left(\widetilde{\eta}^{l}\right)^{-1}$ is the impedance tensor of the $l$ th scattered wave, and $\mathbf{H}_{\mathrm{sc}}^{l m}(b)$ is the tangential magnetic field at the outer interface $r=b$. Applying the evolution operator $\Omega_{a}^{r}$, the electromagnetic field in the shell takes the form

$$
\left(\begin{array}{c}
\mathbf{H}_{\mathrm{sh}}(\mathbf{r}) \\
\mathbf{E}_{\mathrm{sh}}(\mathbf{r})
\end{array}\right)=\sum_{l=0}^{\infty} \sum_{m=-l}^{l}\left(\begin{array}{cc}
F_{l m} & 0 \\
0 & F_{l m}
\end{array}\right) V_{\mathrm{sh}}^{l}(r) \Omega_{a}^{r}\left(\begin{array}{c}
I \\
\Gamma_{1}^{l}(a)
\end{array}\right) \mathbf{H}_{1}^{l m}(a),
$$

where $\Gamma_{1}^{l}=\zeta_{1}^{l}\left(\eta_{1}^{l}\right)^{-1}$ is the impedance tensor of the $l$ th wave inside the inner sphere (region 1 ), and $\mathbf{H}_{1}^{l m}(a)$ is the tangential magnetic field at the inner interface of the shell $r=a$.

By projecting the fields onto the outer interface $r=b$ and integrating over the angles $\theta$ and $\varphi$ with the help of orthogonality condition equation (4), we derive the boundary conditions

$$
\mathbf{W}_{\mathrm{inc}}^{l m}+\left(\begin{array}{c}
I \\
\widetilde{\Gamma}^{l}(b)
\end{array}\right) \mathbf{H}_{\mathrm{sc}}^{l m}(b)=\Omega_{a}^{b}\left(\begin{array}{c}
I \\
\Gamma_{1}^{l}(a)
\end{array}\right) \mathbf{H}_{1}^{l m}(a)
$$

where

$$
\mathbf{W}_{\mathrm{inc}}^{l m}=\int_{0}^{\pi} \int_{0}^{2 \pi}\left(\begin{array}{c}
F_{l m}^{+}(\theta, \varphi) I \mathbf{H}_{\mathrm{inc}}(b, \theta, \varphi) \\
F_{l m}^{+}(\theta, \varphi) I \mathbf{E}_{\mathrm{inc}}(b, \theta, \varphi)
\end{array}\right) \sin \theta \mathrm{d} \theta \mathrm{d} \varphi .
$$

Equation (21) represents the system of four linear equations for four components of the vectors $\mathbf{H}_{\mathrm{sc}}^{l m}$ and $\mathbf{H}_{1}^{l m}$. Finally, one can derive the amplitude of the scattered electromagnetic field (see Appendix)

$$
\begin{aligned}
\mathbf{H}_{\mathrm{sc}}^{l m}(b)= & -\left\{\left[\Gamma_{1}^{l}(a)-I\right] \Omega_{b}^{a}\left[\begin{array}{c}
I \\
\tilde{\Gamma}^{l}(b)
\end{array}\right]\right\}^{-1} \\
& \times\left\{\left[\Gamma_{1}^{l}(a)-I\right] \Omega_{b}^{a} \mathbf{W}_{\mathrm{inc}}^{l m}\right\} .
\end{aligned}
$$

The scattered field in far zone can be characterized by the differential cross section (power radiated to $\mathbf{e}_{r}$ direction per solid angle $d o$ )

$$
\frac{d \sigma}{d o}=r^{2} \frac{\left|\mathbf{H}_{\mathrm{sc}}(\mathbf{r})\right|^{2}}{\left|\mathbf{H}_{\mathrm{inc}}(\mathbf{r})\right|^{2}}
$$

In our notations, the differential cross section averaged over the azimuthal angle $\varphi$ (over polarizations) normalized by the geometrical cross section $\sigma_{g}=\pi b^{2}$ takes the form

$$
\begin{aligned}
& \frac{d \sigma}{\sigma_{g} \sin \theta \mathrm{d} \theta} \\
& \quad=\frac{1}{\sigma_{g}\left|\mathbf{H}_{\mathrm{inc}}\right|^{2}} \sum_{m=-\infty}^{\infty}\left|\sum_{l=|m|}^{\infty} i^{-l-1} F_{l m}(\theta, 0) \tilde{\eta}_{l}^{-1}(b) \mathbf{H}_{\mathrm{sc}}^{l m}(b)\right|^{2} .
\end{aligned}
$$

From the point of view of the scattering theory, it is straightforward to define a cloak as one specially matched layer that provides zero scattering for arbitrary materials inside. In [21] zero scattering was proven analytically for the Pendry's spherical cloak. Here, we have proposed a more general scattering algorithm for radially anisotropic materials, which is useful in studying the scattering of spherical cloaks based on complex (e.g., high-order, nonlinear, etc.) transformations. From the proposed scattering theorem, we 
can determine the invisibility condition (zero scattering) specified by the condition $\mathbf{H}_{\mathrm{sc}}^{l m}(b)=0$, which in turn can be rewritten using Eq. (23) as follows

$$
\left[\Gamma_{1}^{l}(a)-I\right] \Omega_{b}^{a} \mathbf{W}_{\text {inc }}^{l m}=0 .
$$

Arbitrary incident electromagnetic field $\mathbf{W}_{\text {inc }}^{l m}$ can be excluded from this expression. In fact, zero scattering can be obtained for the trivial situation: electromagnetic field is scattered by a "virtual" air sphere at radius $b$. This assumption can be presented in the form analogous to Eq. (26):

$$
\left[\Gamma_{0}^{l}(b)-I\right] \mathbf{W}_{\text {inc }}^{l m}=0,
$$

where $\Gamma_{0}^{l}$ is the impedance tensor of the $l$ th wave in the virtual air sphere. Hence, we have the relation

$$
\left[\Gamma_{1}^{l}(a)-I\right] \Omega_{b}^{a}=\left[\Gamma_{0}^{l}(b)-I\right] .
$$

Impedance tensor $\Gamma_{1}^{l}$ contains material parameters $\varepsilon^{(1)}$ and $\mu^{(1)}$ of the sphere inside the cloaking shell. At the same time, zero scattering should be held for arbitrary $\varepsilon^{(1)}$ and $\mu^{(1)}$ of the inner core. It implies that the partial derivative of Eq. (28) with respect to $\varepsilon^{(1)}$ needs to be zero for arbitrary $\varepsilon^{(1)}$ to satisfy the zero scattering condition. Note that only the impedance tensor $\Gamma_{1}^{l}$ contains $\varepsilon^{(1)}$, and therefore, the right-hand side of Eq. (28) vanishes after the differentiation, which results in $\frac{\partial\left(\Gamma_{1}^{l}(a)-I\right)}{\partial \varepsilon^{(1)}} \Omega_{b}^{a}$ $+\left[\Gamma_{1}^{l}(a)-I\right]\left(\partial \Omega_{b}^{a} / \partial \varepsilon^{(1)}\right)=\left(\frac{\partial \Gamma_{1}^{l}}{\partial \varepsilon^{(1)}} 0\right) \Omega_{b}^{a}=\frac{\partial \Gamma_{1}^{l}}{\partial \varepsilon^{(1)}}\left(\begin{array}{ll}I & 0\end{array}\right) \Omega_{b}^{a}=0$. It is now straightforward that we need the relation to be satisfied, i.e., $\left(\begin{array}{ll}I & 0\end{array}\right) \Omega_{b}^{a}=0$.

By multiplying this equation by $\Gamma_{1}^{l}(a)$ and subtracting it from Eq. (28), we arrive at the equation: (0 $I) \Omega_{b}^{a}$ $=\left[-\Gamma_{0}^{l}(b) I\right]$, which does not contain the material parameters of the inner sphere. Finally, we derive the evolution operator of the cloaking layer,

$$
\Omega_{b}^{a}=\left(\begin{array}{cc}
0 & 0 \\
-\Gamma_{0}^{l}(b) & I
\end{array}\right) .
$$

This condition defines the cloak and can be satisfied for the specially chosen evolution operator $\Omega_{b}^{a}$ of the cloaking shell. The evolution operator is the degenerate block matrix, whose inverse matrix is not defined. It should be noted that relation equation (29) is independent on the material of the inner sphere. On the other hand, the derived relation connects the wave solutions in the cloak (evolution operator $\Omega_{b}^{a}$ ) with wave solutions in the "equivalent" homogeneous air sphere (impedance tensor $\Gamma_{0}^{l}$ ). Therefore, it effectively performs the coordinate transformation for the solutions but not for the material parameters as usual.

Substitution of the cloaking condition $\mathbf{H}_{\mathrm{sc}}^{l m}(b)=0$ into Eq. (21) results in

$$
\Omega_{b}^{a} \mathbf{W}_{\mathrm{inc}}^{l m}=\left(\begin{array}{c}
I \\
\Gamma_{1}^{l}(a)
\end{array}\right) \mathbf{H}_{1}^{l m}(a) .
$$

From Eq. (29), it becomes clear that $\mathbf{H}_{1}^{l m}(a)=0$ and $\mathbf{E}_{1}^{l m}(a)$ $\equiv \Gamma_{1}^{l}(a) \mathbf{H}_{1}^{l m}(a)=0$. Thus one may conclude that both electric and magnetic fields equal zero at the boundary $r=a$, and therefore, there is no field at any spatial point inside the inner sphere. In the cloaking shell, the fields equal zero at the inner boundary $r=a$ (owing to the continuity of the tangential fields) and equal incident fields at the outer boundary $r=b$. Then, the field inside the cloak must obey [see Eq. (20)]

$$
\left(\begin{array}{l}
\mathbf{H}_{\mathrm{sh}}(\mathbf{r}) \\
\mathbf{E}_{\mathrm{sh}}(\mathbf{r})
\end{array}\right)=\sum_{l=0}^{\infty} \sum_{m=-l}^{l}\left(\begin{array}{cc}
F_{l m} & 0 \\
0 & F_{l m}
\end{array}\right) V_{\mathrm{sh}}^{l}(r) \Omega_{b}^{r} \mathbf{W}_{\mathrm{inc}}^{l m} .
$$

\section{NONLINEAR TRANSFORMATION BASED SPHERICAL CLOAKS}

Now, let us consider a class of the NTB spherical cloak, whose electromagnetic (EM) interaction can be characterized by the proposed scattering theorem. Figure 1 can be regarded as the compressed space $(r)$ from the original space $\left(r^{\prime}\right)$, i.e., an air sphere $0<r^{\prime}<b$. We propose a nonlinear transformation function

$$
r^{\prime}=\frac{b^{x+1}}{(b-a)^{x}}\left(1-\frac{a}{r}\right)^{x}
$$

which obviously satisfies the transformation (when $r=a, r^{\prime}$ $=0$, and when $r=b, r^{\prime}=b$ ). The value of " $x$ " is a factor to control the nonlinearity degree in the transformation, which can be arbitrary from 0 to $\infty$.

Due to the invariance of Maxwell's equation under coordinate transformations from the original space to transformed space, the parameters $(\overline{\boldsymbol{\varepsilon}}, \overline{\boldsymbol{\mu}})$ in the shell of Fig. 1 can be expressed in terms of those parameters in the original space, i.e., $\overline{\boldsymbol{\varepsilon}}^{\prime}=1$ and $\overline{\boldsymbol{\mu}}^{\prime}=1$,

$$
\overline{\boldsymbol{\varepsilon}}=A A^{T} / \operatorname{det}(A), \quad \overline{\boldsymbol{\mu}}=A A^{T} / \operatorname{det}(A),
$$

where $A$ is the Jacobian matrix with elements $A_{i j}=\partial r_{i} / \partial r_{j}^{\prime}$.

One can see that the proposed prescribed function equation [Eq. (32)] is only dependent on radial position $r$. Then it is easy to find that the Jacobian matrix is diagonal, and Eq. (33) can thus be rewritten as

$$
\overline{\boldsymbol{\varepsilon}}=\overline{\boldsymbol{\mu}}=\operatorname{diag}\left[\lambda_{r}^{2}, \lambda_{\theta}^{2}, \lambda_{\phi}^{2}\right] / \lambda_{r} \lambda_{\theta} \lambda_{\phi}=\operatorname{diag}\left[\frac{\lambda_{r}}{\lambda_{\theta} \lambda_{\phi}}, \frac{\lambda_{\theta}}{\lambda_{r} \lambda_{\phi}}, \frac{\lambda_{\phi}}{\lambda_{r} \lambda_{\theta}}\right],
$$

where the principal stretches of the Jacobian matrix are

$$
\lambda_{r}=\frac{\partial r}{\partial r^{\prime}}=\frac{(b-a)^{x} r^{x+1}}{x a b^{x+1}(r-a)^{x-1}}, \quad \lambda_{\theta}=\lambda_{\phi}=\frac{r}{r^{\prime}}=\frac{(b-a)^{x} r^{x+1}}{b^{x+1}(r-a)^{x}} .
$$

Finally, one can obtain the parameters of the NTB cloak $(a<r<b)$ in Fig. 1

$$
\begin{gathered}
\varepsilon_{r}=\mu_{r}=\frac{b^{x+1}(r-a)^{x+1}}{x a(b-a)^{x} r^{x+1}}, \\
\varepsilon_{\theta}=\mu_{\theta}=\varepsilon_{\phi}=\mu_{\phi}=\frac{x a b^{x+1}(r-a)^{x-1}}{(b-a)^{x} r^{x+1}} .
\end{gathered}
$$

Such ideal NTB spherical cloak is difficult to be fabricated in practice. However, to some extent, it can be alleviated by dividing the inhomogeneous cloak shell into $N$ ho- 


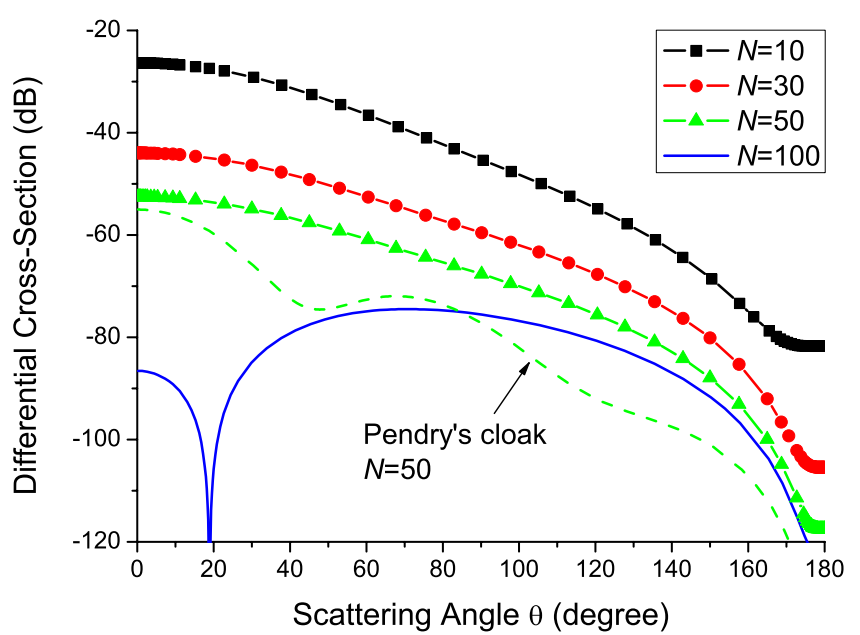

FIG. 2. (Color online) Differential cross section $d \sigma /\left(\sigma_{g} \sin \theta d \theta\right)$ of the NTB spherical cloak $(x=1)$ for different number of the layers $N$ dividing the inhomogeneous coating. Parameters: $\varepsilon^{(1)}=1.45^{2}, \mu^{(1)}=1.0, k_{0} a=\pi$, and $k_{0} b=2 \pi$.

mogeneous multilayers. The case for cylindrical cloaks has been studied and it shows that only several optimized layers can achieve the invisibility [33]. Here, the optimization is out of the scope of this paper. Our paper is to reveal some NTB spherical cloaks, which provides better invisibility performance than Pendry's classic one, based on the proposed general scattering theory. The realistic NTB cloaks can be produced using sputtering techniques so that a number of discrete layers should be applied over the spherical core. To demonstrate the capability of the proposed spherical cloaks, we present the differential cross sections normalized by the geometric cross section of the cloak [see Eq. (25)].

In Fig. 2, we analyze the dependence on the total number of the layers $N$ dividing the cloaking shell. The increase in the layer number gives rise to more accurate approximation of the original inhomogeneous model in Fig. 1, and the decrease of the scattering cross section is expected with the increase of the number $N$. If one uses the present scattering method with $N=50$ to divide both Pendry's linear cloak and a specific NTB cloak at $x=1$, the forward scattering is approximately the same, but over the whole range of scattering angles Pendry's cloak presents better invisibility. The following discussion will address the importance of this nonlinear factor $x$ in beating the classic linear cloak.

The parameter $x$ is a convenient tool to control the quality of the NTB spherical cloak. We assume the number of homogeneous sublayers $N=50$ for all following simulations. In Fig. 3 the differential cross sections at different $x$ are demonstrated. If $x$ is less than unity, the cross section is inversely proportional to $x$, which is not desired in the sense of invisibility. For NTB spherical cloaks with $x<1$, the cloaking performance is degraded due to the abrupt increase of the transverse dielectric permittivity $\varepsilon_{t}$ near the inner interface $r=a$ of the clad (see Fig. 4).

The abrupt change of the material parameters is undesirable not only for invisibility performance but also for the realization point of view. The radial dielectric permittivity behaves in a similarly monotonic way for all values of $x$. The

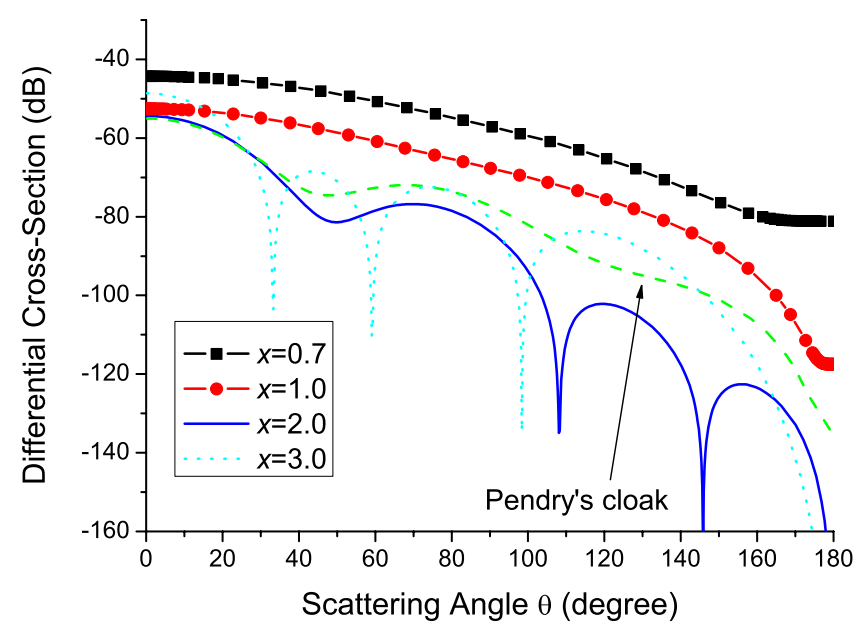

FIG. 3. (Color online) Differential cross section of the nonlinear cloak with different parameters $x$. Parameters: $\varepsilon^{(1)}=1.45^{2}, \mu^{(1)}$ $=1.0, k_{0} a=\pi, k_{0} b=2 \pi$, and $N=50$.

dependence appears to be mainly linear for small parameters $x$. The dependence of the transverse dielectric permittivity is more complicated. One particular NTB cloak is realized at the parameter $x=2$ when the transverse permittivity in the cloak becomes nonmonotonic and eventually returns to $\varepsilon_{t}$ $=2$ at $r=b$, which provides even lower cross section over whole observation angles than the Pendry's cloak does. If we compare the dielectric permittivities of the proposed NTB cloak with that of Pendry's cloak (Fig. 4), it can be noted that the dependence of radial permittivity $\varepsilon_{r}$ are still quite close to each other in the cloak region. However, one may ask whether $x=2$ is the only choice or not. In Fig. 5, it gives the answer that, in the sense of total cross section, there is a range of $x$ in which the proposed NTB spherical cloak outperforms the classic linear spherical cloak. When " $x$ " increases and jumps out of this optimal region, the cloaking effects compared with Pendry's cloak are degraded, which

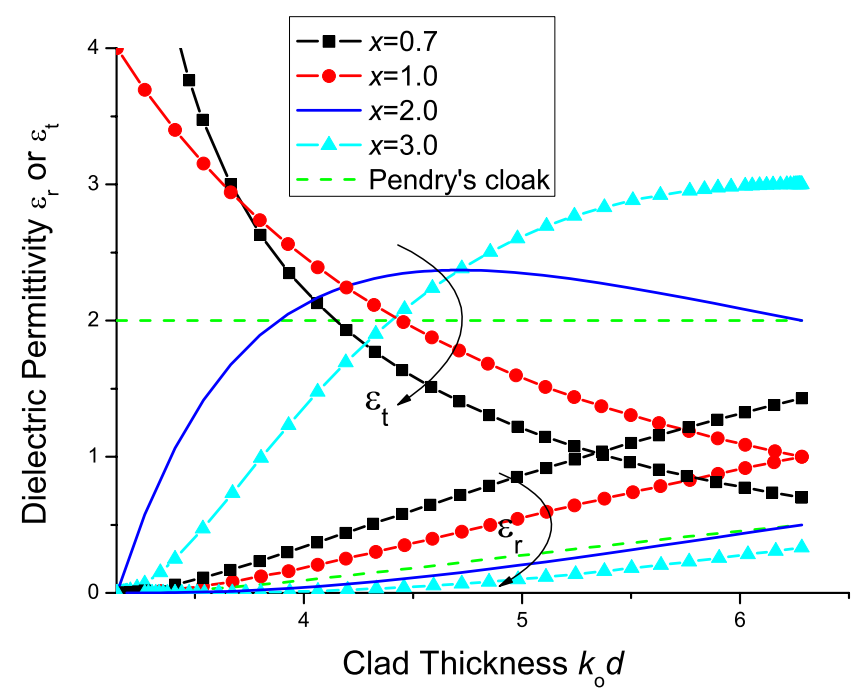

FIG. 4. (Color online) Radial $\varepsilon_{r}$ and transverse $\varepsilon_{t}$ dielectric permittivities of the cloaking shell with different parameters $x$. Cloak is extended from $k_{0} a=\pi$ to $k_{0} b=2 \pi$. 


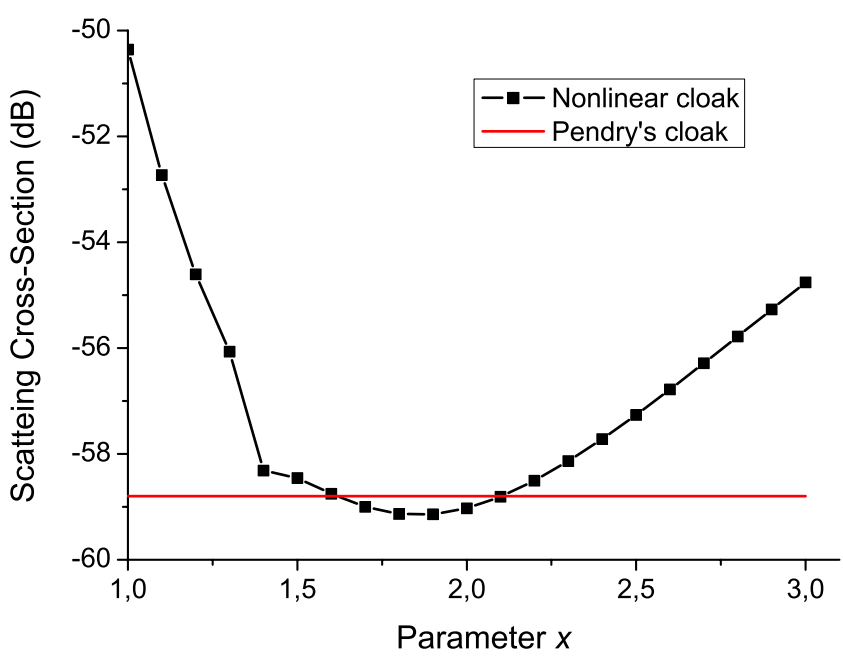

FIG. 5. (Color online) Scattering cross section versus $x$ for NTB spherical cloaks. Parameters: $\varepsilon^{(1)}=1.45^{2}, \mu^{(1)}=1.0, k_{0} a=\pi, k_{0} b$ $=2 \pi$, and $N=50$. The range of $x$ where scattering cross section (SCS) is lower than that of Pendry's spherical cloak is the optimal region of $x$ for desired NTB spherical cloaks.

can be verified by the bistatic cross section of $x=1$ and $x$ $=3$ in Fig. 3 .

Furthermore, we investigate how the transverse permittivity $\varepsilon_{t}$ varies near the optimal region of $x$ in Fig. 5. From Fig. 6 , it can be observed that: (1) when $x$ is slightly above one, the requirement of $\varepsilon_{t}$ near the inner boundary $r=a$ drops significantly compared to those curves whose $x$ is smaller than one in Fig. 5; (2) when $x$ becomes larger and larger, the $\varepsilon_{t}$ at the outer boundary $r=b$ turns to be more deviated from that value of its corresponding $\varepsilon_{r}$; (3) when $x$ falls into the optimal region, those curves of transverse permittivities are nonmonotonic along the radial direction in the cloaking shell, and their maxima and overall values of $\varepsilon_{t}$ are smaller than those whose $x$ becomes further smaller or larger. These explain why there exist an optimal region for $x$ where the total scattering cross section can be lower than Pendry's clas-

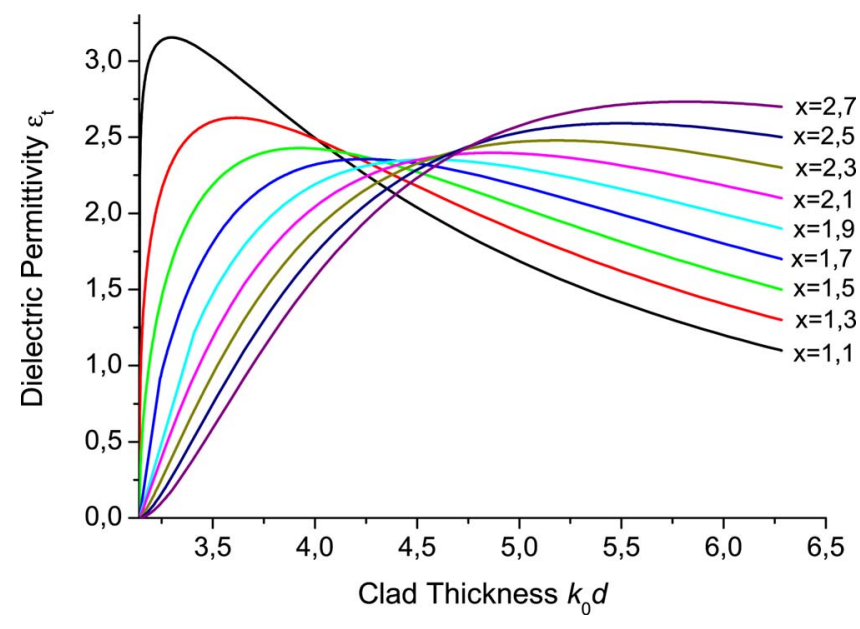

FIG. 6. (Color online) Variance of transverse permittivity $\varepsilon_{t}$ along the radial direction in the region of the cloaking shell $(a<r$ $<b)$ under different values of $x$ near the optimal range as shown in Fig. 5. Parameters: $k_{0} a=\pi$ and $k_{0} b=2 \pi$.

sic one. Also, it provides us another way to predict whether a certain $x$ for a NTB spherical cloak is optimal or not.

Now we continue to study the dependence of its invisibility upon the ratio $b / a$ of the particular NTB spherical cloak with $x=2$, which is discretized into $N=50$ layers. We keep the inner radius $a$ unchanged. In Fig. 7(a), different ratios of $b / a$ are considered. Compared with the other three values of $b / a$, it seems that $b / a=2$ provides the best cloaking effects at nearly all angles (except for the angle at $52^{\circ}$ ) for the $x$ $=2$ NTB cloak. Another interesting finding is that: when $b / a>2$, the cross section will be higher than that of $b / a$ $=2$ over the whole range of angles; when $b / a \rightarrow 1$, although the angle-averaged cross section will still be higher but at certain angles, its cross section could be lower than that of $b / a=2$. From the view of total scattering, it is important to consider how the ratio $b / a$ should be selected for $x=2$ NTB cloak so as to provide improved cloaking. In Fig. 7(b), one can clearly see the optimal domain of $b / a$ in which the cross
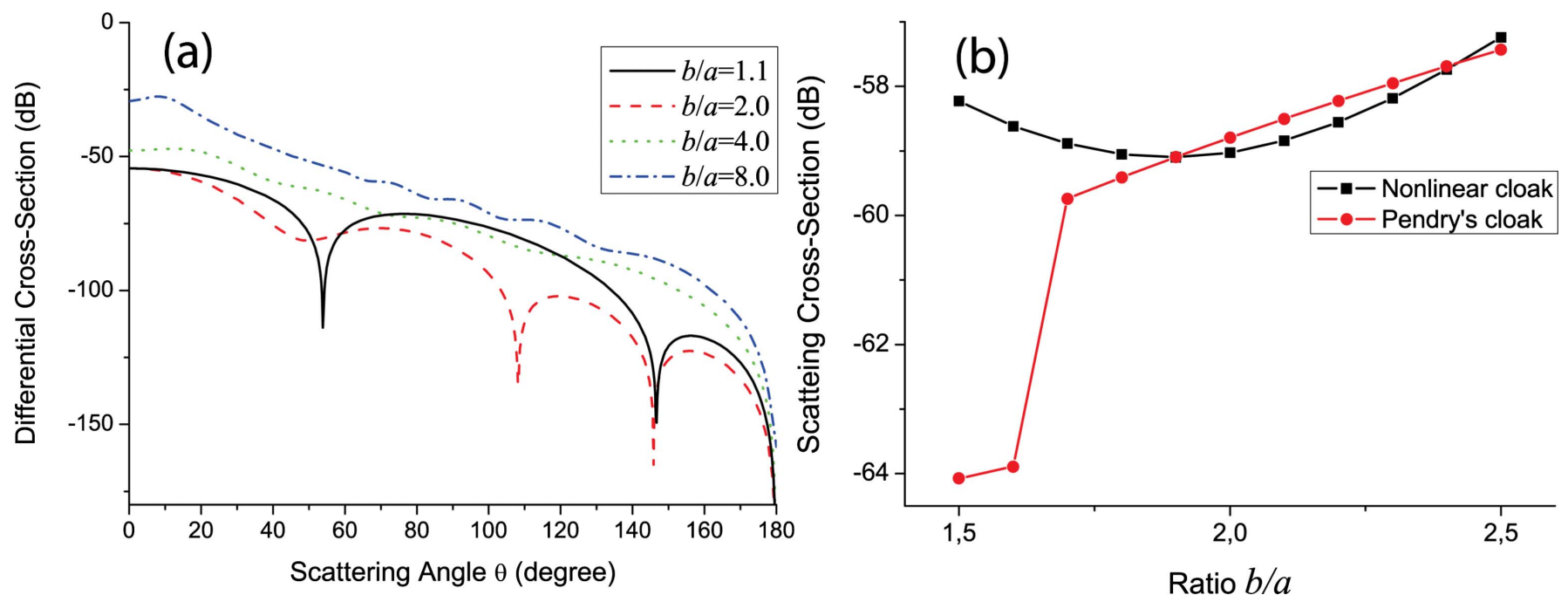

FIG. 7. (Color online) The role of core-shell ratio $b / a$ in the cloaking improvement for $x=2$ NTB spherical cloak: (a) differential cross section versus angle at selected ratios; (b) scattering cross section versus ratio $b / a$. Parameters: $\varepsilon^{(1)}=1.45^{2}, \mu^{(1)}=1.0, k_{0} a=\pi$, and $N=50$. 


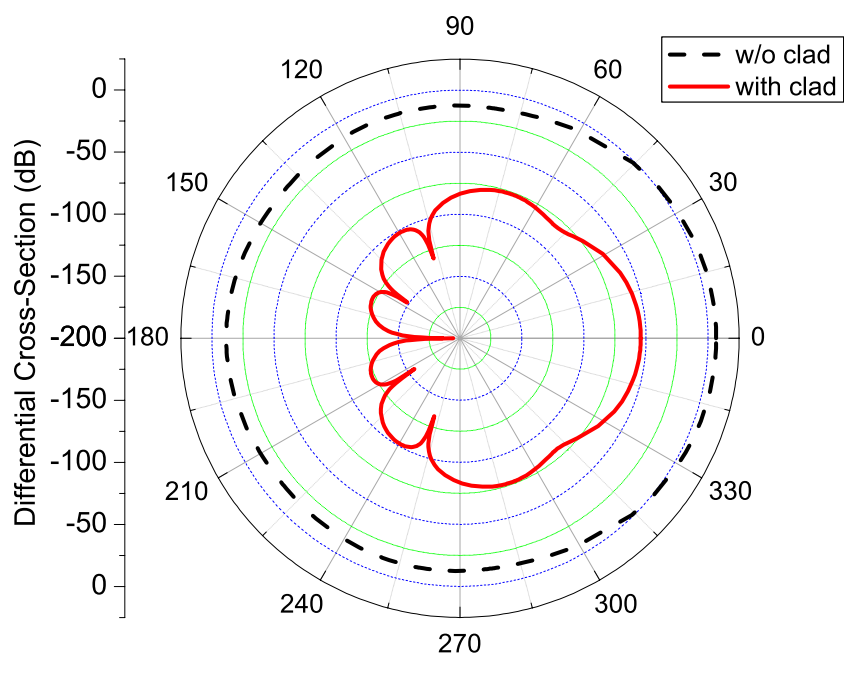

FIG. 8. (Color online) Differential cross section of a glass sphere with and without cloaking shell. Parameters: $\varepsilon^{(1)}=1.45^{2}$, $\mu^{(1)}=1.0, k_{0} a=\pi, k_{0} b=2 \pi, x=2$, and $N=50$.

section is smaller than Pendry's spherical cloak. Certainly, all values $x$ within the desired region for the purpose of improved cloaking in Fig. 5 have their corresponding domain of optimal $b / a$.

Let us compare the scattering diagrams for both a bare glass sphere and a cloaked glass sphere. We take the clad in the form of the nonlinear cloak with parameter $x=2$. From Fig. 8, we see that the cloaking shell noticeably reduces the scattering. Another general property of the cloaks, i.e., the exactly diminished backscattering, is also present in the figure. In what follows, we consider their respective near-field wave interactions, which correspond to far-field results in Fig. 8.

The near-field perturbation of the cloaked and noncloaked glass particles is demonstrated in Fig. 9. Comparing Fig. 9(a) with Fig. 9(b), the invisibility performance is well pronounced. In Fig. 9(a) the EM wave travels only through the clad and takes near zero values in the vicinity of the inner radius $a$. The field does not enter the glass core; therefore, it does not matter which material is situated therein. If the cloak is less ideal than that shown in the figure, the incident field will be scattered by the spherical particle and will penetrate the glass core, i.e., the object becomes visible.

\section{CONCLUSION}

We have studied the manifold of the nonlinear cloaks differing in parameter $x$. Since there is no closed-form solution
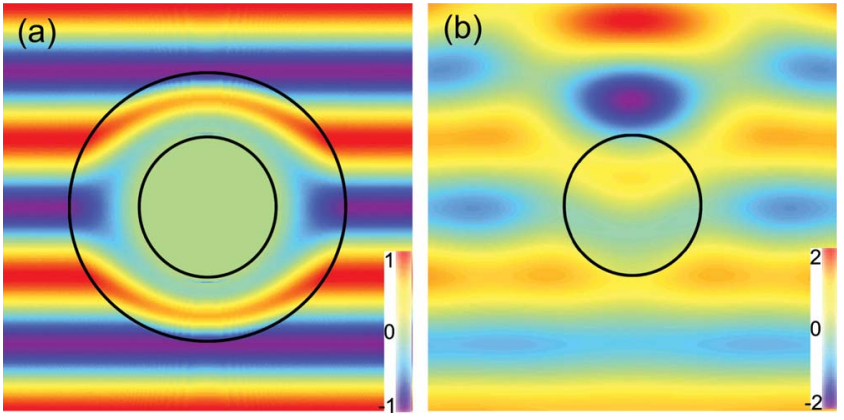

FIG. 9. (Color online) Real part of the electric field on $x$ - $z$ plane scattered (a) by the cloaking shell gathered round the glass core and (b) by the glass core itself. Parameters: $\varepsilon^{(1)}=1.45^{2}, \mu^{(1)}=1.0, k_{0} a$ $=\pi, k_{0} b=2 \pi, x=2$, and $N=50$.

for the proposed NTB cloaks, an approximate model by replacing an inhomogeneous shell with homogeneous spherical layers has been numerically analyzed, with the help of the proposed scattering algorithm for multilayered rotationally anisotropic shells. The general cloaking condition was derived from the scattering algorithm, which is in contrast to the method of coordinate transform. We have also demonstrated that better approximate spherical cloaks can be realized by properly choosing parameters $x$ and $b / a$. In practical applications, such a class of NTB spherical cloaks can provide improved invisibility performance.

\section{APPENDIX}

In order to exclude the constant vector $\mathbf{H}_{1}^{l m}$, one should multiply Eq. (21) by $\Omega_{b}^{a}=\left(\Omega_{a}^{b}\right)^{-1}$,

$$
\Omega_{b}^{a} \mathbf{W}_{\mathrm{inc}}^{l m}+\Omega_{b}^{a}\left(\begin{array}{c}
I \\
\widetilde{\Gamma}^{l}(b)
\end{array}\right) \mathbf{H}_{\mathrm{sc}}^{l m}(b)=\left(\begin{array}{c}
I \\
\Gamma_{1}^{l}(a)
\end{array}\right) \mathbf{H}_{1}^{l m}(a) .
$$

Then Eq. (A1) is further multiplied by the block matrix $\left[\Gamma_{1}^{l}(a)-I\right]$, and the right-hand side vanishes,

$$
\left[\Gamma_{1}^{l}(a)-I\right] \Omega_{b}^{a} \mathbf{W}_{\mathrm{inc}}^{l m}+\left(\Gamma_{1}^{l}(a)-I\right) \Omega_{b}^{a}\left(\begin{array}{c}
I \\
\tilde{\Gamma}^{l}(b)
\end{array}\right) \mathbf{H}_{\mathrm{sc}}^{l m}(b)=0
$$

On the other hand, vector $\mathbf{H}_{1}^{l m}$ can be obtained in a similar way.
[1] J. B. Pendry, D. Schurig, and D. R. Smith, Science 312, 1780 (2006).

[2] U. Leonhardt, Science 312, 1777 (2006).

[3] G. W. Milton, M. Briane, and J. R. Willis, New J. Phys. 8, 248 (2006).

[4] W. Cai, U. K. Chettiar, A. V. Kildishev, and V. M. Shalaev,
Nat. Photonics 1, 224 (2007).

[5] S. A. Cummer, B. I. Popa, D. Schurig, D. R. Smith, and J. Pendry, Phys. Rev. E 74, 036621 (2006).

[6] Z. Ruan, M. Yan, C. W. Neff, and M. Qiu, Phys. Rev. Lett. 99, 113903 (2007).

[7] F. Zolla et al., Opt. Lett. 32, 1069 (2007). 
[8] D. Schurig et al., Science 314, 977 (2006).

[9] M. Yan, Z. Ruan, and M. Qiu, Phys. Rev. Lett. 99, 233901 (2007).

[10] W. Yan, M. Yan, Z. Ruan, and M. Qiu, J. Opt. Soc. Am. A Opt. Image Sci. Vis 25, 968 (2008).

[11] M. Yan, Z. Ruan, and M. Qiu, Opt. Express 15, 17772 (2007).

[12] W. Cai, U. K. Chettiar, A. V. Kildishev, V. M. Shalaev, and G. W. Milton, Appl. Phys. Lett. 91, 111105 (2007).

[13] I. Gallina, G. Castaldi, and V. Galdi, Microwave Opt. Technol. Lett. 50, 3186 (2008).

[14] C. W. Qiu and B. S. Luk'yanchuk, J. Opt. Soc. Am. A Opt. Image Sci. Vis 25, 1623 (2008).

[15] L. W. Cai and J. Sanchez-Dehesa, New J. Phys. 9, 450 (2007).

[16] Y. Huang, Y. Feng, and T. Jiang, Opt. Express 15, 11133 (2007).

[17] J. Zhang, Y. Luo, H. Chen, and B.-I. Wu, J. Opt. Soc. Am. B 25, 1776 (2008).

[18] W. X. Jiang, J. Y. Chin, Z. Li, Q. Cheng, R. Liu, and T. J. Cui, Phys. Rev. E 77, 066607 (2008).

[19] H. Ma, S. Qu, Z. Xu, and J. Wang, Phys. Rev. E 78, 036608 (2008).

[20] A. Greenleaf, Y. Kurylev, M. Lassas, and G. Uhlmann, Commun. Math. Phys. 275, 749 (2007).

[21] H. Chen, B. I. Wu, B. Zhang, and J. A. Kong, Phys. Rev. Lett.
99, 063903 (2007).

[22] B. Zhang, H. Chen, B. I. Wu, and J. A. Kong, Phys. Rev. Lett. 100, 063904 (2008).

[23] L. Gao, T. H. Fung, K. W. Yu, and C. W. Qiu, Phys. Rev. E 78 , 046609 (2008).

[24] A. Alu and N. Engheta, Opt. Express 15, 7578 (2007).

[25] A. Alu and N. Engheta, J. Opt. A, Pure Appl. Opt. 10, 093002 (2008).

[26] C. W. Qiu, Li Hu, X. F. Xu, and Y. J. Feng, Phys. Rev. E 79, 047602 (2009).

[27] T. Tyc and U. Leonhardt, New J. Phys. 10, 115038 (2008).

[28] C. W. Qiu, L. W. Li, T. S. Yeo, and S. Zouhdi, Phys. Rev. E 75, 026609 (2007).

[29] J. D. Jackson, Classical Electrodynamics (Wiley, New York, 1998), Chaps. 3 and 9.

[30] F. I. Fedorov, Theory of Gyrotropy (Nauka i Tehnika, Minsk, 1976).

[31] C. W. Qiu, S. Zouhdi, and A. Razek, IEEE Trans. Antennas Propag. 55, 3515 (2007).

[32] A. V. Novitsky and L. M. Barkovsky, Phys. Rev. A 79, 033821 (2009).

[33] B. I. Popa and S. A. Cummer, Phys. Rev. A 79, 023806 (2009). 